[3] Fujimoto M, Hamaguchi Y, Kaji K et al. Myositis-specific anti-155/140 autoantibodies target transcription intermediary factor 1 family proteins. Arthritis Rheum. 2012;64(2):513-22.

[4] Rothwell S, Chinoy H, Lamb JA et al. Focused HLA analysis in Caucasians with myositis identifies significant associations with autoantibody subgroups. Ann Rheum Dis. 2019;78(7):996-1002.

Disclosure of Interests: None declared

DOI: 10.1136/annrheumdis-2021-eular.964

\section{POS0289 CANCER RISK IN IMMUNOSUPPRESSED SCLERODERMA PATIENTS: A PROPENSITY SCORE MATCHING ANALYSIS}

L. Verardi ${ }^{1}$, E. De Lorenzis ${ }^{1}$, G. Natalello ${ }^{1}$, L. Gigante ${ }^{1}$, M. A. D'agostino ${ }^{1}$, S. L. Bosello' ${ }^{1}{ }^{1}$ Fondazione Policlinico Universitario A. Gemelli IRCCS - Catholic University of the Sacred Heart, Rheumatology Unit, Rome, Italy

Background: An increased incidence of cancer in patients with systemic sclerosis (SSc) is well-established ${ }^{1}$. Current knowledge about the onco-transforming power of immunosuppressive treatments in both non-rheumatological and rheumatological pathologies, suggests an increased incidence of hematological and solid neoplasms ${ }^{2}$. Evidences on the possible role of the immunosuppressants in the onset of cancer during SSc are lacking.

Objectives: To evaluate the incidence of malignancies in SSc patients exposed to immunosuppressive therapy.

Methods: The incidence of neoplasia in a cohort of 600 patients with SSc was evaluated retrospectively. Patients diagnosed with malignancy within 36 months from SSc onset were excluded from the analysis since suspected for paraneoplastic form. Patients exposed to methotrexate, cyclophosphamide, azathioprine and mycophenolate were confronted with a group comparable for age at onset, sex, disease variant, autoantibodies and exposure to other disease-specific therapies, using propensity score matching analysis. The considered follow-up was between the clinical onset and the diagnosis of cancer or the last available visit or the twenty-fifth year of illness.

Results: The analysis was carried out on 526 patients observed for an average period of $12.1 \pm 6.0$ years (males $9.5 \%$, age at onset $49.0 \pm 15.3$ years); $24.9 \%$ had diffuse cutaneous variant of the disease and $39.0 \%$ and $34.8 \%$ were respectively positive for anti-centromere and anti-Scl70 antibodies. During the follow-up, $19.0 \%$ of patients were exposed to cyclophosphamide, $15.3 \%$ to azathioprine, $14.4 \%$ to methotrexate and $11.6 \%$ to mycophenolate mofetil. Sixty-five cancer diagnoses were made after the 36th month from onset (incidence 1.02: 100 patients/year), comprising 16 mammary cancers, 12 cancers of the gastro-intestinal tract, 11 cancers of the head-neck area, 10 cancers of the lungs, 9 cases of skin-cancer, 5 haematological malignancies and 1 brain tumour. The incidence of cancers did not differ in relation to treatment with cyclophosphamide $\left(X^{2}=0.001, p=0.961\right)$, azathioprine $\left(X^{2}=2.141, p=0.143\right)$, mycophenolate mofetil $\left(X^{2}=0.001, p=0.993\right)$ or methotrexate $\left(X^{2}=0.920, p=0.337\right.$ ) (Figure 1). Conclusion: Our data are consistent with an increased incidence of neoplasms in the course of SSc, with a rate that appears almost doubled compared to the general Italian population with similar sex and age distribution (0.55: 100 patients/year; Italian
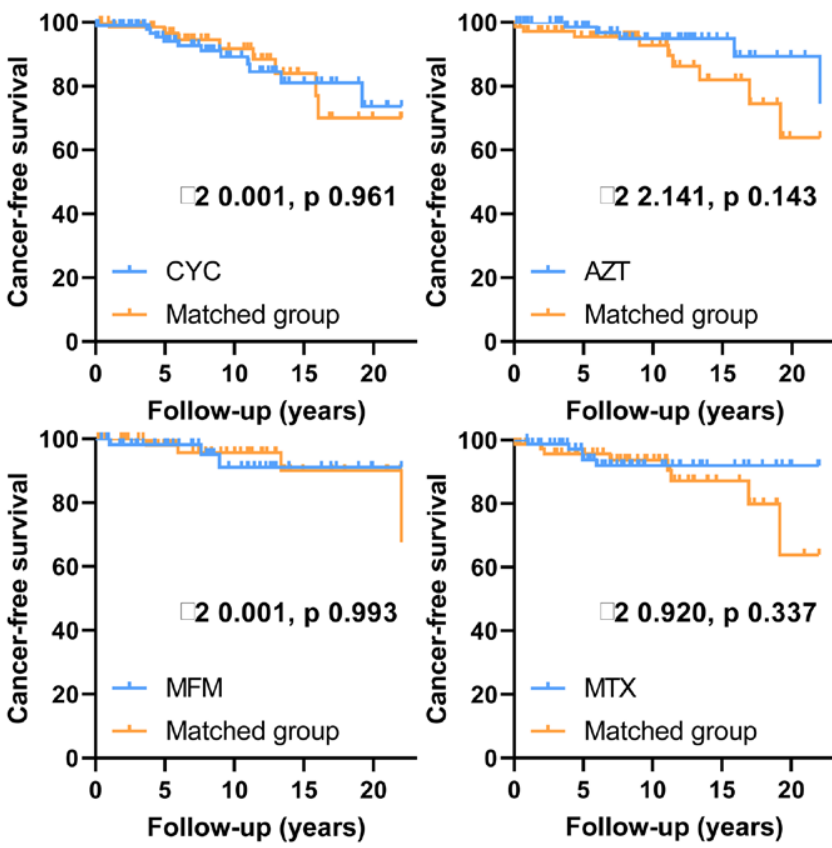

Association of Tumor Registries data ${ }^{3}$ ). In our SSc cohort this risk is independent of exposure to immunosuppressive drugs commonly used for the treatment of scleroderma disease.

REFERENCES:

[1] Akira O et al. Cancer Incidence in Systemic Sclerosis: Meta-Analysis of Population-Based Cohort Studies. Arthritis \& Rheumatism. 2013

[2] Zitvogel $L$ et al. Cancer despite immunosurveillance: immunoselection and immunosubversion. Nat Rev Immunol. 2006

[3] Registri Tumori di popolazione in Italia: la Banca Dati AIRTUM (Associazione Italiana Registri Tumori). 2020

Disclosure of Interests: None declared

DOI: 10.1136/annrheumdis-2021-eular.2701

\section{POS0290 PREDICTING RISK FACTORS OF MALT LYMPHOMA IN SJÖGREN'S SYNDROME}

L. Chatzis ${ }^{1}$, V. Pezoulas ${ }^{2}$, A. Goules ${ }^{1}$, I. Stergiou' ${ }^{1}$, C. Mavragani ${ }^{3}$, H.

M. Moutsopoulos ${ }^{4}$, M. Voulgarelis ${ }^{1}$, D. Fotiadis ${ }^{2}$, A. Tzioufas ${ }^{1} .{ }^{1}$ School of

Medicine, National and Kapodistrian University of Athens, Pathophysiology,

Ag thoma 15, Greece; ${ }^{2}$ University of loannina, Unit of Medical Technology

and Intelligent Information Systems, Ioannina, Greece; ${ }^{3}$ School of Medicine,

National and Kapodistrian University of Athens, Physiology, Athens, Greece

${ }^{4}$ Academy of Athens, Chair Medical Sciences/Immunology, Greece, Athens,

Greece

Background: Primary Sjögren Syndrome (SS) is a slowly progressive systemic autoimmune disease complicated by lymphoma, with mucosa associated lymphoid tissue (MALT) type being the most common lymphoma form. Several predictors related to pSS associated lymphomas have been described, but there are no studies focusing on specific risk factors for the MALT histologic subtype. Objectives: To identify predictors at SS diagnosis for MALT lymphoma development in pSS patients using simple clinical features.

Methods: From 815 SS patients of a single center fulfilling the 2016 ACR/EULAR criteria, those with subsequent development of MALT Iymphoma according to the 2016 WHO classification were identified and matched in 1:2 ratio, with non-lymphoma SS control patients according to age, disease duration from SS diagnosis and gender. Lymphoma patients diagnosed within a year from SS diagnosis were excluded from the current study. Clinical, laboratory, histologic data as well as the ESSDAI scores at the time of SS diagnosis were recorded and compared between lymphoma and non-lymphoma patients. Independent lymphoma predictors were identified by a data driven Fast Correlation Based Feature selection (FCBF)/Logistic Regression (LR) algorithm.

Results: A unified dataset of 57 MALT lymphoma patients and 114 non lymphoma controls along with 39 features/variables was generated. The median age of SS diagnosis and the disease duration from SS diagnosis to lymphoma diagnosis (lymphoma group) or last follow up (control group) was 50,5 years old (range 25-77) and 7 years (range 0 - 30) for the control group and 50 years old (range 24-70) and 8 years (range 1 -30) for the lymphoma group, respectively. MALT lymphoma patients presented more frequently with palpable purpura (23,2\% vs $5,3 \% p=0,001)$, cryoglobulinemia $(30,2 \%$ vs $1,6 \% p<0,0001)$, low $C 4$ serum levels $(62,9 \%$ vs $32,1 \% p=0,0003)$, rheumatoid factor $(76,9 \%$ vs $56,1 \%$ $\mathrm{p}=0,01)$, anti $\mathrm{La} / \mathrm{SSB}$ antibodies $(33,9 \%$ vs $50,8 \% \mathrm{p}=0,049)$ and higher median ESSDAI score ( 5 vs 2, $p<0,0001$ ). In contrast, autoimmune thyroiditis was more prevalent in controls $(48,2 \%$ vs $18,6 \%, p=0,004)$. The FCBF/LR model revealed cryoglobulinemia $(p=0,03)$ and ESSDAI at SS Diagnosis $(p<0,001)$ as the only independent lymphoma predictors.

Conclusion: MALT is the predominant pSS related lymphoproliferative histologic type, associated with systemic disease activity and vasculitic manifestations a SS diagnosis. Cryoglobulinemia and ESSDAI score were proven independent risk factors for MALT Iymphoma development.

Table 1. An FCBF-based multivariable logistic regression analysis results for investigating risk factors for MALT Iymphoma development

\begin{tabular}{lccccc}
\hline Prominent feature & Regression coefficient & Odds ratio & p-value & $\mathrm{Cl}$ low & $\mathrm{Cl}$ upper \\
\hline Cryoglobulinemia & 1.67 & 5.342 & $0.033^{*}$ & 1.18 & 24.327 \\
Total ESSDAl at diagnosis & 0.28 & 1.318 & $<0.001^{\star}$ & 1.208 & 1.439 \\
Kidney involvement & 0.07 & 1.069 & 0.5 & 0.101 & 0.351
\end{tabular}

$\bullet 0.05$ (95\% confidence interval). The rest of the features that participated in the analysis include the following: Palpable purpura, Low C4, Salivary gland enlargement, Lacrimal gland enlargement, ANA Titers, RF, Focus score at Sjögren diagnosis, PNS involvement, Anti-La, Disease duration from SS onset to SS diagnosis, Neutrophils $<1500$, Autoimmune thyroiditis, Lung involvement - interstitial disease Type, Lymphocytes $<1000$, Lymphadenopathy fixed, Arthralgias, Dry eyes, Raynaud, Gender, Age at Sjögren diagnosis, Dry mouth, Aca, $\mathrm{Hb}<12.5 \mathrm{~g} / \mathrm{dL}$, ANA, Disease duration from SS Diagnosis to Lymphoma diagnosis or last follow up, Anti-Ro, Arthritis, WBC $<4000 / \mu \mathrm{L}$, Lung involvement bronchocentric disease, Interstitial renal disease, PLTs $<100.000 / \mu \mathrm{L}$, Liver involvement-PBC, Liver involvement-autoimmune hepatitis, CNS involvement. $\cdot A U C=0.78$ 
Disclosure of Interests: None declared

DOI: 10.1136/annrheumdis-2021-eular.2260

\section{POS0291 THE IMPACT OF DRUG INDUCED AND OTHER RHEUMATIC MUSCULOSKELETAL DISORDERS (MD) ON THE QUALITY OF LIFE (QOL) OF CANCER PATIENTS RECEIVED ANTICANCER DRUG TREATMENT}

A. Koltakova ${ }^{1}$, A. Lila ${ }^{2}$, L. P. Ananyeva ${ }^{1}$, A. Fedenko ${ }^{3} .{ }^{1}$ V. A. Nasonova Research Institute of Rheumatology, Laboratory of Microcirculation and Inflammation, Moscow, Russian Federation; ${ }^{2}$ V. A. Nasonova Research Institute of Rheumatology, Adminisrtation, Moscow, Russian Federation; ${ }^{3}$ National Medical Research Radiological Centre of the Ministry of Health of the Russian Federation, Department of Drug Treatment of Cancer, Moscow, Russian Federation

Background: Pts with cancer may have MD that can be caused by neoplastic/ paraneoplastic disease, rheumatic diseases or be induced by anticancer drug treatment. There is no data about MD influence on the QoL of cancer patients. The EORTC QoL questionnaire (QLQ)-C30 is a valid questionnaire designed to assess different aspects (Global health $(\mathrm{GH})$, Functional (FS) and symptoms (SS) scales) that define the QoL of cancer patients [1]

Objectives: The objective of the study was to assess the impact of drug induced and other types of MD on the QoL of cancer patients that received anticancer drug treatment by using of EORTC QLQ-C30 v3.0.

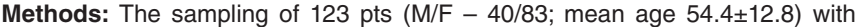
breast $(32,5 \%)$, gastrointestinal $(17 \%)$, ovary $(8 \%)$, lung $(7 \%)$ and other cancer was observed by rheumatologist in the oncology outpatient clinic. All pts received anticancer drug treatment: chemotherapy (104 pts), target therapy (16 pts) checkpoint-inhibitors (14 pts), hormone therapy (13 pts) in different combinations. $102(82.9 \%)$ of $123 p t s$ had MD include arthritis (12 pts), synovitis (5 pts), arthralgia (66 pts), periarthritis (34 pts), osteodynia (13 pts). There were 58 pts (group $1 ; \mathrm{M} / \mathrm{F}-14 / 44$; mean age $52.5 \pm 12.2$ ) with anticancer drug treatment induced MD and 44 pts (group 2; M/F - 16/27; mean age 57.6 \pm 13.5 ) with other type of MD include 26 pts with skeletal metastasis. The were 21 pts (group 3; M/F - 10/11;

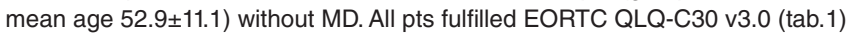

Table 1. The median [Q1;Q3] of results of $\mathrm{GH}, \mathrm{SS}$ and SS of EORTC QLQ-C30

\begin{tabular}{lllll}
\hline Scale & \multicolumn{1}{c}{ Subscale } & \multicolumn{1}{c}{ Group1 } & \multicolumn{1}{c}{ Group2 } & \multicolumn{1}{c}{ Group3 } \\
\hline GH & & $58.3[50 ; 58]$ & $58.3[41.7 ; 83.3]$ & $50[50 ; 66.7]$ \\
FS $^{*}$ & Physical functioning & $73.3[60 ; 86.7]$ & $73.3[66.7 ; 86.7]$ & $86.7[80 ; 93]$ \\
& Role functioning & $66.7[66.7 ; 100]$ & $83.3[50 ; 100]$ & $100[83 ; 100]$ \\
& Emotional functioning & $83.3[66.7 ; 100]$ & $75[66.7 ; 91.7]$ & $91.6[83.3 ; 100]$ \\
& Social functioning & $83.3[66.7 ; 100]$ & $83.3[50 ; 100]$ & $100[83.3 ; 100]$ \\
SS $^{*}$ & Pain & $33.3[0 ; 50]$ & $16.7[0 ; 33.3]$ & $0[0 ; 16.7]$
\end{tabular}

*There are only the scores that had got a statistical difference between the groups.

Kruskal-Wallis $\mathrm{H}$ and post-hoc (Dwass-Steel-Critchlow-Fligner (DSCF) pairwise comparisons) tests for data analysis were performed.

Results: A Kruskal-Wallis $\mathrm{H}$ test has shown a statistically significant difference in physical $(\alpha 2(2)=7.54 ; p=0.023)$, role $(\alpha 2(2)=9.87 ; p=0.007)$, emotion $(\alpha 2(2)=7.69$; $p=0.021)$ functioning and pain $(\chi 2(2)=8.44 ; p=0.015)$ scores between the different groups. A post-hoc test with DSCF pairwise comparisons of median has shown a statistically significant difference between 1 and 3 groups $(W=3.904$; $\mathrm{p}=0.016)$ for physical functioning, between 2 and 3 groups $(\mathrm{W}=3.35 ; \mathrm{p}=0.004)$ for role functioning, between 2 and 3 groups $(W=4.03 ; p=0.012)$ for emotional functioning, between 1 and 3 groups $(W=-3.97 ; p=0.014)$ for pain scale.

Conclusion: The study has shown that MD associated with anticancer drug treatment adversely affected the QoL of cancer patients received anticancer drug treatment by reducing a physical functioning and by increasing pain scores. Presence of other types of MD adversely affect the QoL by reducing emotional and role functioning. REFERENCES:

[1] Aaronson NK,et al.The European Organization for Research and Treatment of

Cancer QLQ-C30: a quality-of-life instrument for use in international clinical trials in oncology. J Natl Cancer Inst.1993;85(5):365-376. doi:10.1093/jnci/85.5.365 Disclosure of Interests: None declared

DOI: 10.1136/annrheumdis-2021-eular.2286

\section{POS0292 \\ OSTEOPOROSIS IN BREAST CANCER PATIENTS ON AROMATASE INHIBITORS THERAPY: ABOUT 200 CASES}

R. Assadi ${ }^{1}$, K. Nassar ${ }^{1}$, S. Janani ${ }^{1} .{ }^{1} \mathrm{CHU}$ ibn rochd, Rheumatology, Casablanca, Morocco
Background: Breast cancer is the most common cancer affecting women both before and after the menopause. Aromatase inhibitors (Als) used as adjuvant therapy cause bone loss and increase the risk of osteoporosis (OP) (1).

Objectives: To assess the bone status and the frequency of OP in breast cancer patients using Als.

Methods: We conducted a retrospective study in the rheumatology department, over 5 years (2016-2020)

Inclusion criteria: patients followed for breast neoplasm in oncology department on Als therapy and referred to rheumatology department for bone evaluation. All of these patients underwent an evaluation of bone mineral density (BMD) and phosphocalcic assessment.

Exclusion criteria: patients treated by another type of hormone therapy and having other risk factors for OP.

Results: 200 breast cancer women were enrolled for the study, 92 patients $(46 \%)$ were treated with Als, the average age was 58.22 years ( 41 - 75 years) with an average age of discovery of the breast cancer of 46.75 years and the average time between the start of Als therapy and the diagnosis of OP was 21.6 months. the characteristics of the patients are summed up in Table 1.

$97 \%$ of patients were postmenopausal and $38 \%$ of them had menopause secondary to treatment.

In Als users, 85 patients (92.3\%) were osteoporotic, and $11 \%$ had bone fractures. In regard to osteodensitometry measurement, lumbar spine was the most affected site ( $88 \%$ ) with mean T score of -2.98 and mean BMD of 0.854 followed by femoral neck (17\%) with mean T score of -2.8 and mean BMD of 0.740 and total hip (14\%) with mean $T$ score of -3.10 and mean BMD of 0.497 .

The cancer was metastatic in $15.18 \%$ patients, $75 \%$ of the group had bone metastasis and $25 \%$ had visceral metastasis.

The phosphocalcic status of the osteoporotic patients was: mean calcemia: $92.83 \mathrm{mg} / \mathrm{I}$, mean calciuria: $131.8 \mathrm{mg} / 24 \mathrm{~h}$, mean phosphatemia: $48.09 \mathrm{mg} / \mathrm{I}$, mean 25 OH Vit D level: $19.78 \mathrm{ng} / \mathrm{ml}$, mean PTH: $79 \mathrm{pg} / \mathrm{ml}$.

Osteoporotic patients were treated with bisphosphonates, $60 \%$ women had received Alendronate, $17 \%$ Risedronate and $12 \%$ Zoledronate in addition to dietary measures and correction of calcium and vitamin D deficiency.

Conclusion: Als are correlated with a high risk of OP and fractures in $30 \%$ of patients (2), the frequency of OP in our series is estimated at $42.5 \%$.

Assessment of bone status and OP clinical risk factors should be systematic in all breast cancer patients receiving adjuvant Als therapy.

Bisphosphonates appear to be beneficial in treating secondary OP, in preventing bone fractures, and in reducing the incidence of breast cancer bone metastases. REFERENCES:

[1] Aromatase Inhibitor-Associated Bone Loss Clinical Considerations P Shubham, L. Shapiro Drugs 2008; 68 (18): 2591-2600

[2] Aromatase inhibitor-associated bone loss and its management with bisphosphonates in patients with breast cancer M.Bauer, J. Bryce, P.Hadji Breast Cancer. 2012; 4: 91-101

Table 1. Characteristics of osteoporotic patients using Als therapy

\begin{tabular}{ll}
\hline & Results \\
\hline Total of patients & $92(46 \%)$ \\
Mean age & 58.22 years \\
Mean age of breast cancer diagnosis & 46.75 years \\
Delay between Als therapy and OP & 21.6 months \\
Post menopausal patients & $97 \%$ \\
Menopausis due to Als & $38 \%$ \\
Osteoporotic patients & $85(92.3 \%)$ \\
Fractures & $11 \%$ \\
Sites of OP in osteodensitometry & \\
lumbar spine & $88 \%$ \\
femoral neck & $17 \%$ \\
total hip & $14 \%$ \\
Cancer metastasis & $15.18 \%$ \\
Bone & $75 \%$ \\
Visceral & $25 \%$ \\
Phosphocalcic status (mean values) & \\
calcemia & $92.83 \mathrm{mg} / \mathrm{l}$ \\
calciuria & $131.8 \mathrm{mg} / 24 \mathrm{~h}$ \\
phosphatémie & $48.09 \mathrm{mg} / \mathrm{l}$ \\
25 OH vitaminD & $19.78 \mathrm{ng} / \mathrm{ml}$ \\
PTH & $79 \mathrm{pg} / \mathrm{ml}$ \\
Biphosphanates therapy & \\
Alendronate & $60 \%$ \\
Risedronate & $17 \%$ \\
Zoledronate & $12 \%$ \\
\hline &
\end{tabular}

Disclosure of Interests: None declared DOI: 10.1136/annrheumdis-2021-eular.2886 